\title{
On the Promo Film of Chengdu's Urban Image Construction Applying for the 2025 World Games Based on Multimodal Metaphor
}

\author{
Wu Yanmin, Peng Yi
}

School of Foreign Studies, Yangtze University, Hubei, P.R. China

\begin{abstract}
As a way of thinking and cognition, metaphor not only exists in language modality, but also exists in non-linguistic modality such as image, voice and gesture which form multimodal discourses by their interactions. Based on the theory of multimodal metaphor, this paper interprets the cognitive construction of the promo film of the 2025 World Games "Gather in Chengdu, Cheer the World on", in which it analyzes the urban image and provides a new perspective for the analysis of urban image.
\end{abstract}

Keywords-metaphor, multimodal discourse, urban image, promo film, cognition.

\section{INTRODUCTION}

The construction of urban image depends on its active communication to some extent. Promo film, as an important means to disseminate urban image, can reflect its comprehensive strengths and influences, as well as historical culture, national spirits and values. Therefore, to host the World Games, Chengdu needs to publicize itself to the world. Promo film is intuitive, enjoyable and convenient to spread because of the summary and sorting of urban culture and the construction of a unique urban image with specific visual symbols. In the era of modal diversity, multimodal discourse has become an integral part of life. Compared with pure text, multimodal discourse composed of language, images, sound, text and gesture can convey information, exchange feelings and publicize urban image more vividly.

Multimodal metaphor provides a new perspective for the analysis of multimodal discourse. Foreign research achievements on multimodal metaphor mainly come from the collected papers Multimodal Metaphors (2009), of Forceville and Urios-Aparisi, which mainly focused on advertisement and cartoon (Zhao, 2011). Domestic research on multimodal metaphor started late, focusing on deconstruction of multimodal text, such as advertisement, cartoons on current politics and icons (Zhang \& Yao, 2019). In addition, a few scholars have studied national image promo film from the perspective of multimodal metaphor (Pan \& Zhang, 2013; Yang \& Tang, 2016; Guan, 2018). However, there are few cognitive interpretations of urban promo film discourse from multimodal perspective.

In view of this, from the perspective of multimodal metaphor, this paper takes the image promo film of Chengdu as an example to analyze the cognitive mechanism of multimodal metaphors, so that it can provide a deeper understanding of similar promo films and multimodal metaphors. At the same time, the promo films can help to increase the publicity efforts, establish a good urban image and show the infinite urban charm.

\section{MULTIMODAL METAPHOR THEORY}

Metaphor theory was first proposed in Lakoff \& Johnson's book The Metaphor We Live By (1980) that summarizes the essential characteristics of metaphor which is cognitive. It shows that we can conceptualize the less clearly delineated in terms of the more clearly delineated 
by metaphor, that is, to understand another thing by one thing. It is not only expressed in the language, but also can be extended beyond the range of ordinary literal ways of thinking and talking into the range of what is called figurative, poetic, colorful, or fanciful thought and language. Metaphors do not emerge in a vacuum but base on our own experiences. Metaphor expresses a mapping relationship which makes abstract concepts materialized to deepen our understanding (Lakoff, 1980). Metaphor is a discourse strategy of identity construction, because people use metaphor to think, to explain to others and to organize discourses. The choice of metaphor not only reveals the conceptualization results of communicators, but also reveals their attitudes and values (Sun et al., 2017).

Modality refers to the channel and medium of communication. Forceville (2009) defined it as "a symbolic system that can be interpreted by a specific perceptual process". The research results of life science showed that the living body gradually acquires five different perceptual channels of vision, hearing, smell, taste and touch in the process of evolution, which leads to the production of five modalities of visual modality, auditive modality, olfactory modality, gustatory modality, tactile modality (Zhu, 2007). Later, Forceville subdivided the modality into image symbols, written symbols, verbal symbols, gestures, sounds, music, smells, tastes and contacts for the convenience of research. What's more, Forceville also distinguished between "monomodal metaphor" and "multimodal metaphor". A monomodal metaphor is the metaphor that uses only one modality to represent the target domain and source domain, such as language, image or visual modality. Multimodal metaphor is the metaphor that uses two or more different modalities to represent source and target domains, such as visual and auditory modalities at the same time (Forceville, 2009).

Forceville summarized the advantages of multimodal metaphor into the following four aspects. First, images, sounds, and gestures have direct perceptuality which are not available in language and more refined in expression. Second, multimodal metaphor has more choices in revealing the similarity between the source domain and target domain. Third, multimodal metaphor is more easily recognized and accepted by audiences from different cultural backgrounds because images and sounds are more universal than languages. Fourth, the source domain of multimodal metaphor may have stronger emotional impact than that of pure text metaphor (Forceville, 2008).

\section{DEFINITION OF THE PROMO FILM AND ITS MULTIMODALIZATION}

Guo (2011) defined the promo film as the type of film that the communicator influences the audience through modern media such as images, sounds and words to convey ample information. Urban promo film is used to publicize a city with sounds, images and other means and states the politics, economy, urban construction, culture, history and humanities of cities comprehensively. A high-quality urban promo film will not only enhance the taste and influence of a city, but also attract investors' attention and promote the rapid development of the region.

Zhu (2007) proposed two standards for the recognition of multimodal discourses. One is the number of types involved in modal discourses, making the distinction between monomodal discourse and multimodal discourse. He thought that monomodal discourse only uses one modality, such as listening to music by auditive modality or reading books by visual modality. Multimodal discourse uses two or more modalities simultaneously such as enjoying a dish by olfactory modality, visual modality and gustatory modality. The other is the number of symbolic systems involved. Some discourses involve only one modality, but contain two or more symbolic systems. For example, cartoons involve only visual modality but contain both text and pictures which is also called multimodal discourse.

The promo film, a dynamic multimodal discourse which is a typical multimodal text, expresses the economy, politics, culture and humanity of a city vividly through sounds, words, images, actions and other media.

\section{THE MULTIMODAL METAPHORICAL DISCOURSE MEANING OF THE PROMO FILM "GATHER IN CHENGDU, CHEER THE WORLD ON"}

Multimodal discourse is a phenomenon of communication by means of a various of means and 
symbolic resources such as language, image, sound and action and senses such as hearing, vision and touch (Zhang, 2009). The Promo Film of Chengdu reflects its economy and culture by the metaphorical means, fully showing its advantages. The Promo Film has distinct themes, exquisite pictures and harmonious music which successfully show characteristics and customs of Chengdu and convey the enthusiasm of people with the self-narration of panda.

\subsection{Logo and slogan}

The logo of Chengdu 2025 World Games is displayed with the initials $\mathrm{C}$ of Chengdu and panda's ears (Fig. 1) which stands for five continents, both independent and integrated mutually, meaning that Chengdu will be a bridge of friendship for building a stage of world exchanges to welcome friends from all over the world to gather in Chengdu.
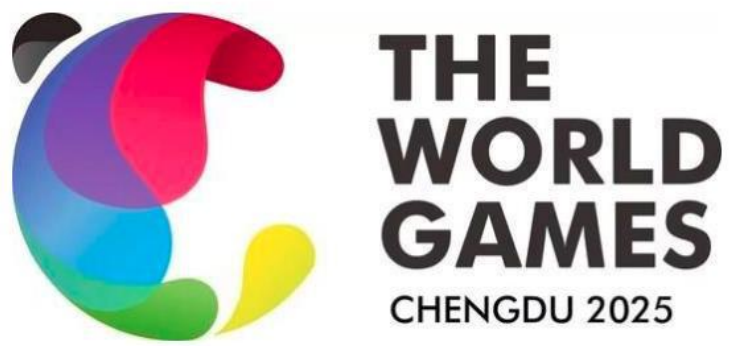

Fig. 1: The logo of the 2025 World Games

Chengdu's slogan, “Gather in Chengdu, Cheer the World on", expresses the desire showing the world a city with ancient cultural traditions and modern charm. The World Games 2025 will be an environmentally friendly, safe, sustainable and successful games that will develop and popularize World Games programs and work together to create a sustainable World Games heritage.

\subsection{Contents of the promo film}

\subsubsection{Anthropomorphic metaphor}

Example one, "Panda is Chengdu's ambassador for the application as host city for the 2025 World Games".

The whole promo film introduces Chengdu by a panda including geographical location, culture, climate, cultural deposits, economy, transportation, the sports events it has hosted and will host. The promo film begins with the panda's self-statement as the ambassador for the 2025 World Games, which shows the anthropomorphic metaphor "Panda is a person" (Fig. 2) and it has human character, feelings and values. Anthropomorphic metaphor is vivid and narrowing the distance between the audience and the target domain.

The promo film gives the panda the human image more often, such as wrestling, playing tug-of-war, playing billiards and dancing which reveals that China attaches importance to the events. The panda, as the national treasure of China, become the ambassador of the World Games, which reflects the enthusiasm of Chengdu's people.

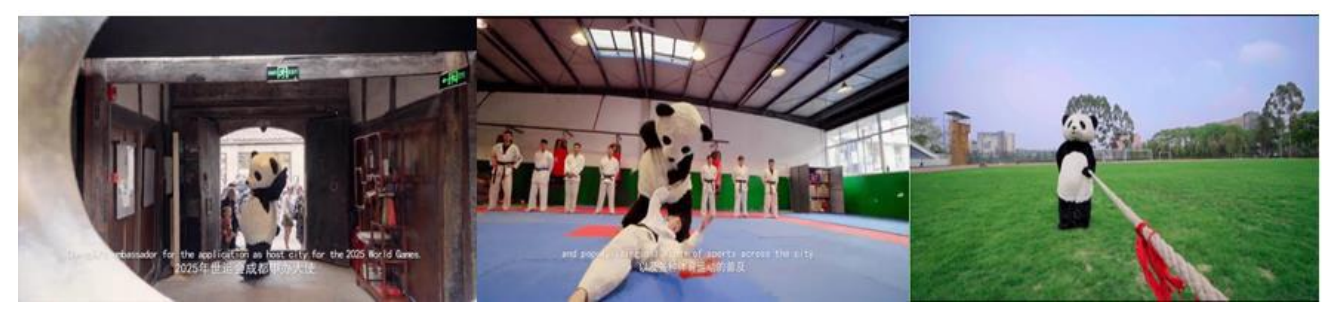

Fig.2: The ambassador of the World Games

Example two, "Chengdu is the happiest city in China".

"Chengdu has the sense of happiness" is an anthropomorphic metaphor, and it endues Chengdu with human psychology (Fig. 3), vividly showing the comfortable environment, convenient transportation, and warm hospitality of Chengdu and facilitating the public to understand and experience Chengdu's urban appearance. Chengdu is also called Lotus city, the land of abundance, and Jinguan city from which we can feel the comfortable environment of Chengdu and it also lays a good foundation for the success of the events. 


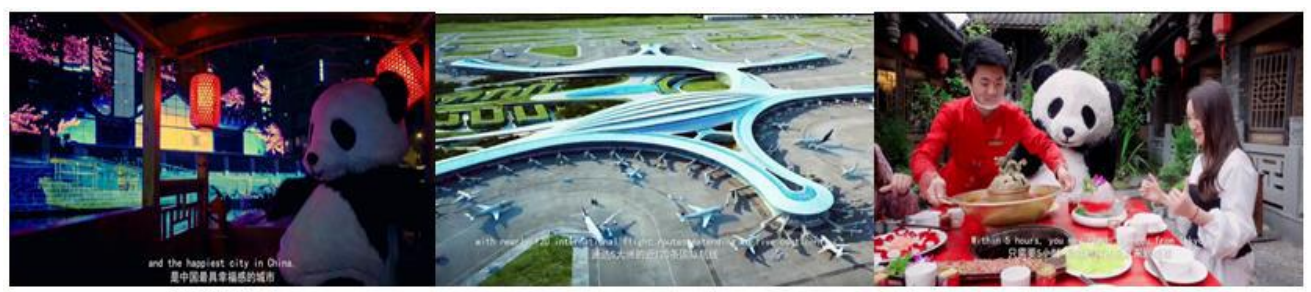

Fig.3: The happiest city

Example three, "But in Chengdu we could coexist with humankind".

Humans, not animals, are responsible for the act of "coexist with humankind"
(Fig. 4). Giving animals the characteristics of human reflects the panda's status as an ambassador and demonstrates the hospitality of people in Chengdu, which indicates the success of Chengdu 2025 World Games.

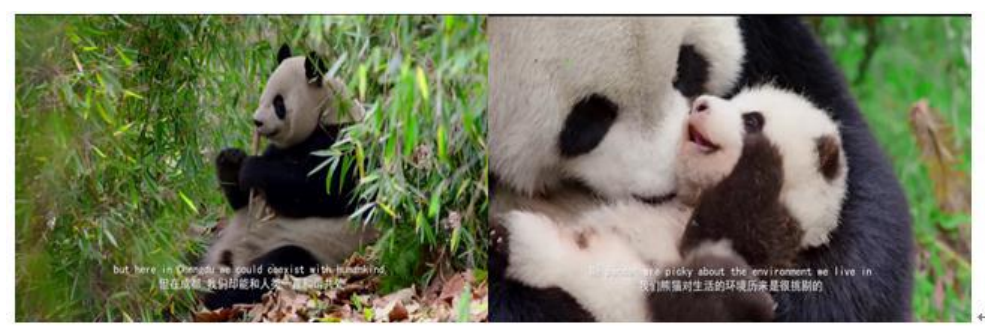

Fig.4: Coexist with humankind

Example four, "Sport has injected this city with life force"!

"City with life force" is an anthropomorphic metaphor (Fig. 5) as cities are compared here to people in sports. Athletes are full of passion which reflects the characteristics of Chengdu' people loving sports.

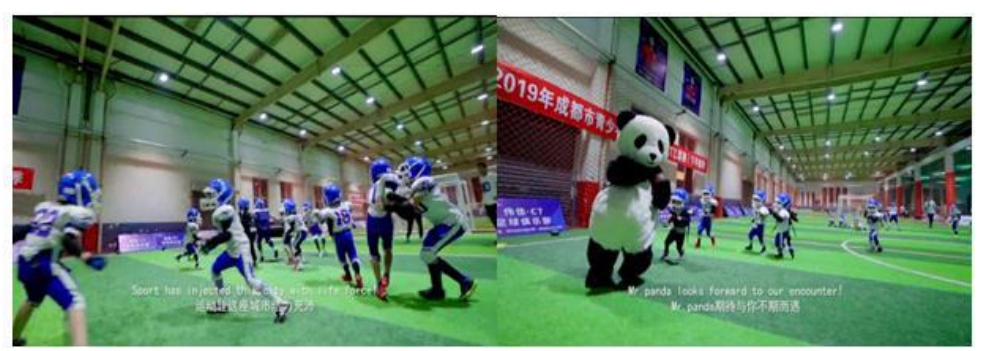

Fig.5: The city with life force

\subsubsection{Journey metaphor}

Journey metaphor is a conceptual metaphor throughout the whole promo film, which powerfully connects all parts of the promo film and conveys the development process and vision of Chengdu from the past, now to the future. The journey has a starting point, direction and destination. Similarly, the development of a city also has its history, direction and goal. This systematic structural metaphor enables the audience to understand the development of a city from the perspective of a familiar ISSN: 2456-7620 journey.

Example five, "Chengdu has 2300 years of history as a city, and is one of the 'ten ancient capitals of China' as well as the birth place of jiaozi - the earliest paper money in the world. Chengdu is at the forefront of China's westward and southward opening-up efforts, one of the 'mega cities' closest to Central Asia and Europe, and ranks third in the nation in the number of foreign consulates".

It is a conceptual metaphor "Chengdu's development 
is a journey" (Fig. 6). The architectural history of Chengdu can be traced back to 2300 years ago which makes Chengdu become one of the "ten ancient capitals of China" with long history of architecture. With the development of economy and culture, the earliest paper money in the world was born in Chengdu.

The long history of Chengdu also has laid a good foundation for its current development, making it the forefront of China's westward and southward opening-up efforts and one of the "mega cities" closest to Central Asia and Europe. The development of Chengdu shown the promo film has aroused people's knowledge about its history and deepened the public's impression on Chengdu adding to the cultural symbols appearing on the screen.
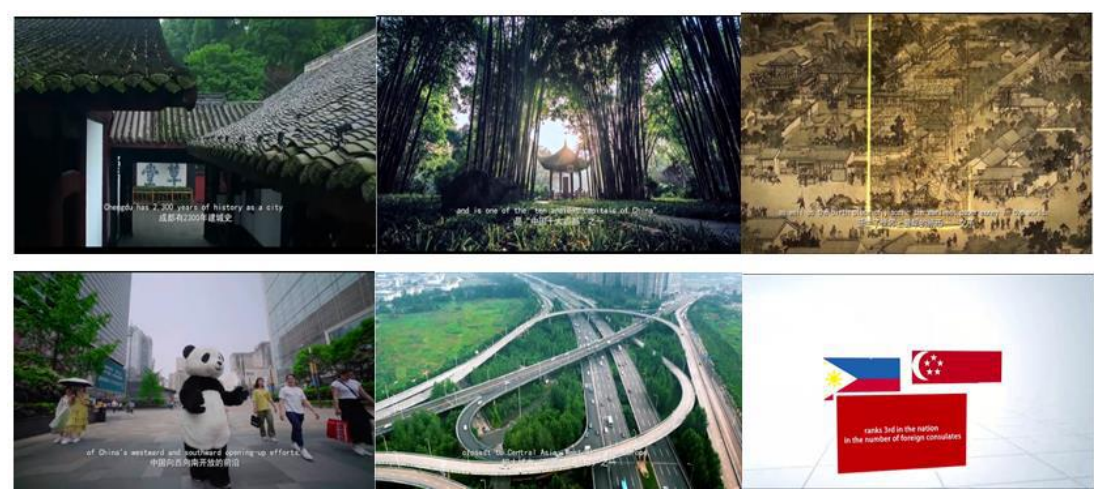

Fig.6: Chengdu's development is a journey

Example six, "At present, Chengdu is dedicated to positioning itself as a world-famous city for sports, and the Chengdu Municiple Government is committed to driving the construction of athletic facilities, promoting the application for hosting international sports compititions, and popularizing all kinds of sports across the city. In the past three years, Chengdu has already played host to more than 160 high-level Chinese sports competitions, and has cooperated with international sports governing bodies, including Federation of Association Football, International
Basketball Federation, International Tennis Federation, Association of Tennis Professionals, International Table Tennis Federation and World Dance Sport Federation among other organizations, hosting 56 international sports competitions".

It is an illustration of "Sports development in Chengdu is a journey" (Fig. 7). The sports picture, cheerful dance, modern city and athletes' love for sports in the promo film convey the development process and vision of Chengdu from the past, now to the future.
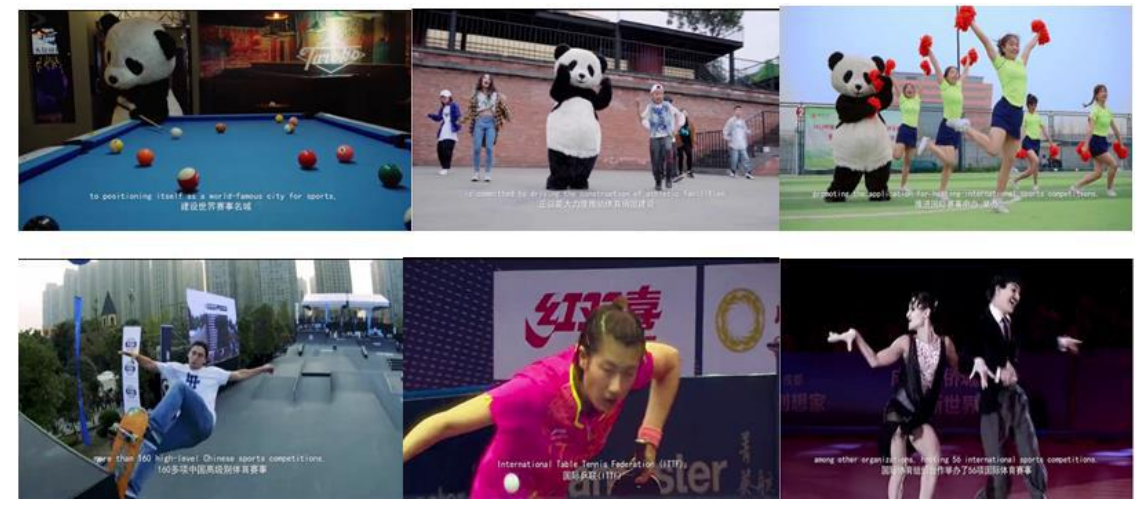

Fig.7: Sports development in Chengdu is a journey 


\subsubsection{Container metaphor}

Container metaphor is the most typical and representative metaphor in ontological metaphor. Human being is an entity independent of the surrounding world and everyone is a container including indoors and outdoors. Peoples often project this concept onto other objects outside the human body, such as houses, jungles, fields, etc., and even some intangible and abstract events, behaviors, activities and states are also seen as a container (Liu, 2013).

Example seven, "Chengdu's new economic vitality is listed at the forefront among Chinese cities".

Chengdu is compared to a container here. Nearly 300 Fortune Global 500 corporates have established a presence in the city (Fig. 8) which contains the ontological metaphor of "New economic vitality is enterprise". Enterprises with economic strength settled in Chengdu which injects new vitality into the development of Chengdu. The steady flow of traffic and the crowd with the happy rhythm in the promo film represent a broad and bright development prospect brought by the new economic vitality.

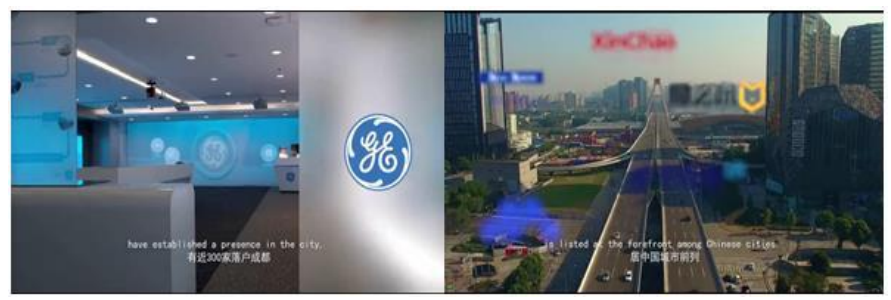

Fig.8: New economic vitality

Example eight, "The ever-present greenway has already assimilated into the daily life of Chengdu-ers"!

Here, life is compared to a container (Fig. 9). The greenway symbolizes a healthy lifestyle. Brisk movements, cheerful rhythm and harmonious music all reflect the spirit of paying attention to health and environmental protection of people, which is consistent with the theme of the World Games.

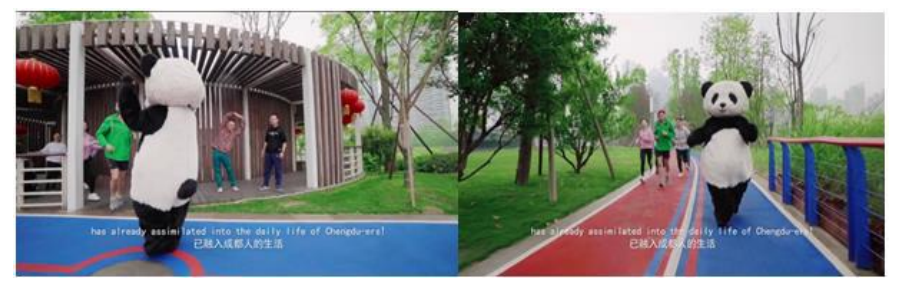

Fig.9: Life is a container

\subsubsection{Orientational metaphor}

Orientational metaphor refers to a series of metaphorical concepts based on spatial location. Spatial orientation originates from the interaction between people and nature such as "up" and "down", "front" and "behind", etc. These specific concepts are used to express emotion, physical condition, quantity, social status and some other abstract concepts in orientational metaphor (Liu, 2013).

Example nine, "Nearly 300 Fortune Global 500 corporates have established a presence in the city. Chengdu's new economic vitality is listed at the forefront among Chinese cities".

"Front" is a direction (Fig. 8). In this promo film, the modern city and convenient transportation are both the performances of the new economic vitality, implying that Chengdu's economic development is outstanding and has a good prospect. 


\subsubsection{Stage metaphor}

The promo film regards the modern high-rise buildings as the background. Actors dressed in costumes, people with happy smiles and food reflect the conceptual metaphor "Chengdu is a big stage" (Fig. 10). This implies that people in Chengdu are putting on a splendid play of life and writing a magnificent chapter in the big stage of modernization construction.

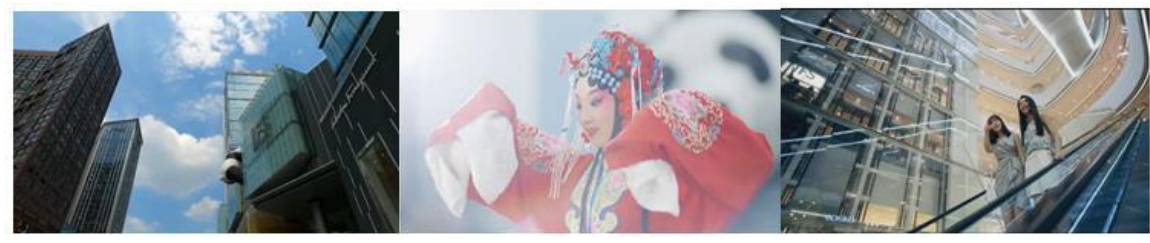

Fig.10: Chengdu is a big stage

\section{COGNITIVE CONSTRUCTION OF MULTIMODAL METAPHORICAL DISCOURSE MEANING}

The promo film of Chengdu 2025 World Games gives people a great visual impact with cheerful rhythm, oral commentary and brisk music, which fully reflects the improvement of Chengdu's economic, political and cultural strength. It expresses Chengdu people's expectation for the World Games guests from all over the world to gather together as happy and harmonious as friends dating.

Each city has its idea of development and values. In the promo film of Chengdu World Games, a city full of vitality and happiness and harmonious animals are anthropomorphic metaphors which is made of athletes with a healthy body, citizens with a healthy lifestyle, convenient transportation and comfortable living environment, outlining the harmonious natural and cultural environment of Chengdu.

The development process of a city is a journey. A journey has its starting point, direction and destination, so does a city. Chengdu has a history of 2300 years of architecture and the birth of the world's earliest paper money, which is the starting point. Chengdu is at the forefront of China's westward and southward opening-up efforts, and the Chengdu Municiple Government is committed to driving the construction of athletic facilities, promoting the application for hosting international sports compititions, and popularizing all kinds of sports across the city, which is the direction. The goal of Chengdu's development is to build itself into a world-famous ISSN: 2456-7620 tournament city. The process of journey is a structural metaphor that expresses the development of Chengdu.

In the promo film, Chengdu is a container to absorb fortune 500 companies which inject new vitality into Chengdu. The new vitality means strong economic strength and indicates the prosperity of Chengdu's economy. Container metaphor refers not only to entity, but also to some invisible and abstract things. The greenways everywhere have become part of Chengdu people's lives. The greenway is an entity, and life is invisible. The greenway, as an ontological metaphor, refers to a healthy lifestyle which shows the livable environment of Chengdu.

"Chengdu's new economic vitality is listed at the forefront among Chinese cities" is a typical orientational metaphor. "Front" is opposite to "behind", and it means excellent and is always associated with something positive. "Forefront" means Chengdu's developed economy and good future. Dance, comedy and high-rise buildings outline the rich cultural deposits and strong economic strength of Chengdu.

In a word, metaphor reflects the way of human brain to know the world. It recognizes unfamiliar, intangible, abstract and rare conceptual domains from familiar, tangible, concrete and common conceptual domains, thus establishing the connection between different conceptual systems (Liu, 2013).

\section{CONCLUSION}

Multimodal metaphor is the latest development of cognitive metaphor theory. The paper makes the 
identification and interpretation of "Gather in Chengdu, Cheer the World on" by the multimodal metaphor. Through synergy of a variety of modals, not only the traditional culture and modern development of Chengdu are completely showed in language, but also the various features of Chengdu are revealed by dynamic modalities including voice, pictures and expression with harmonious beautiful music, thus revealing the cognitive mechanism of multimodal discourse, arousing the emotional resonance and enhancing the favorable impression on city of audience and achieving the aim of publicity. This study, to some extent, makes up for the lack of previous studies on the understanding mechanism of multimodal metaphor, so as to provide relevant references for the publicity of sports events in the future.

\section{REFERENCES}

[1] Forceville, C. (2008). Metaphors in pictures and multimodal representations. In R. W. Gibbs, Jr (Eds.), The Cambridge Handbook of Metaphor (pp. 462-483). Cambridge: Cambridge University Press.

[2] Forceville, C. (2009). Non-verbal and multimodal metaphor in a cognitivist frame-work: agendas for research. In C. Forceville \& E. Urios-Aparisi (Eds.), Multimodal Metaphor (pp. 19-42). Berlin/New York: Mouton de Gruyter.

[3] Guan, S. (2018). The construction of Russian national image by multimodal metaphors - take the Russian national publicity film as an example. Foreign Language Research in Northeast Asia, 6(03), 16-20.

[4] Guo, Y., Wu, X. (2011). The historical source of promo films. Journalism Lover, (04), 100-102.

[5] Lakoff, G., Johnson, M. (1980). Metaphors We Lived By. Chicago: University of Chicago Press.

[6] Liu, R. (2013). Western schools of linguistics. Beijing: Foreign Language Teaching and Research Press.

[7] Pan, Y., Zhang, H. (2013). The cognitive mechanism of multimodal discourses - a case study of the documentary China's national image promotion: perspectives. Foreign Languages Research, (01), 10-19, 112.

[8] Sun, Y., Qian, Y., Ma, T. (2017). The current status and development trends in metaphor studies. Modern Foreign Languages, 40(05), 695-704, 731.

[9] Yang, Y., Tang, Q. (2016). Study on multimodal metonymy and its functions in national image promo film: a case of Russian national image promotion documentary. Journal of Zhejiang Sci-Tech University (Social Sciences Edition), 36(05), 461-467.

[10] Zhang, D. (2009). On a synthetic theoretical framework for multimodal discourse analysis. Foreign Languages in China, 6(01), 24-30.

[11] Zhang, L., Yao, L. (2019). Retrospect and prospect of domestic multimodal metaphor and multimodal metonymy (2011-2018). Journal of Shandong University of Technology (Social Sciences Edition), 35(02), 63-69.

[12] Zhao, X. (2011). New development of conceptual metaphor studies: multimodal metaphor. Foreign Languages Research, (01), 1-10, 112.

[13] Zhu, Y. (2007). Theory and methodology of multimodal discourse analysis. Foreign Language Research, (05), $82-86$ 